\title{
ARTIGO DE REVISÃO Qualidade de vida em cuidadores de idosos portadores de demência de Alzheimer
}

\author{
Quality of life of Alzheimer's disease caregivers' people \\ Juliane dos Anjos de Paula', Francelise Pivetta Roque², Flávio Soares de Araújo3
}

\section{RESUMO}

Objetivo: Realizar revisão sistemática da literatura acerca da QV dos cuidadores de idosos com DA. Métodos: Revisão sistemática de literatura por meio do PubMed e da MedLine entre os anos de 1997 e 2008, utilizando os termos quality of life, caregivers, Alzheimer disease. Resultados: Dezesseis estudos entre 274 publicações iniciais e 39 artigos posteriormente incluídos pelas referências bibliográficas foram avaliados. A QV dos cuidadores dos idosos demenciados, na maioria dos estudos, estava comprometida negativamente. Diversos fatores interferem na QV do cuidador, desde a gravidade da doença até alterações compor-

\section{Palavras-chave}

Qualidade de vida, cuidadores, demência.

\section{Keywords}

Quality of life, caregivers, Alzheimer disease. tamentais que esses idosos possam vir a apresentar. Conclusão: É necessária a realização de mais estudos direcionados a esse tema, a fim de buscar estratégias para melhora da QV desse cuidador.

\section{ABSTRACT}

Objective: Carry out a systematic bibliographycal review about QL of the caregivers of in the elderly with AD. Methods: A systematic bibliographical review about studies indexed on Pubmed and MedLine, from 1997 to 2008, using as key-words the terms: quality of life, caregivers, Alzheimer Disease. Results: Sixteen of the 274 initial articles and 39 additional papers included in a following phase, based on bibliographical references, were evaluated. A negative impairment in QL of the caregivers of demential in the elderly has been documented in most studies. Several factors affect the caregivers' quality of life, such as severity of the patient's illness and behavior disorders of elderly. Conclusion: Futures studies on this theme will be necessary to look for strategies.
1 Faculdade de Medicina da Universidade Estadual de Ciências da Saúde de Alagoas (Uncisal).

2 Faculdade de Fonoaudiologia da Uncisal e Escola Paulista de Medicina da Universidade Federal de São Paulo (EPM/Unifesp). 3 Departamento de Psiquiatria da Faculdade de Medicina da Uncisal, Universidade Federal de Alagoas (Ufal) e Antropologia da EBAF, Israel. Este trabalho foi realizado na Universidade Estadual de Ciências da Saúde de Alagoas (Uncisal). 
A qualidade de vida é definida como a percepção do indivíduo sobre sua posição na vida, no contexto da cultura e do sistema de valores nos quais ele vive e em relação aos seus objetivos, expectativas, padrões e preocupações'. Trata-se de conceito amplo, que abrange a complexidade do construto e inter-relaciona o meio ambiente com os aspectos físicos e psicológicos, o nível de independência, as relações sociais e as crenças pessoais².

O uso das medidas de qualidade de vida é relevante na avaliação de saúde, tanto dentro da perspectiva social quanto individual, sobretudo nos quadros de doenças degenerativas, em que a mensuração da eficácia do tratamento se traduz na qualidade de vida aos anos vividos, diante da ausência de cura. Diante do envelhecimento populacional, tanto dos países desenvolvidos quanto dos em desenvolvimento ${ }^{3}$, o gerenciamento destas doenças torna-se aspecto relevante de saúde pública, não somente ao grande número de indivíduos acometidos como de cuidadores destes indivíduos, já que muitas destas doenças, ao prejudicarem a autonomia e/ou a dependência do portador da doença, tornam necessária a figura de alguém responsável pelos seus cuidados ${ }^{4,5}$.

Entre as doenças neurodegenerativas, uma das mais impactantes é a demência, não somente pela grande ocorrência (5\% a 10\% das pessoas acima de 65 anos, podendo chegar a $47 \%$ dos idosos com mais de 85 anos $^{6}$ ) como também pelos índices de morbidade preocupantes nos cuidadores e/ou familiares dos portadores de demência ${ }^{7,8}$.

O termo "demência" caracteriza síndromes de etiologias diversas, cujo aspecto fundamental é o prejuízo da memória, acompanhado de, pelo menos, uma outra função cognitiva (linguagem, praxia, gnosia ou funções executivas), a ponto de comprometer o funcionamento ocupacional ou social e representar declínio em relação ao nível anteriormente superior de funcionamento 9 . Entre as causas de demência, a doença de Alzheimer é a principal delas, sendo responsável por cerca de 55\% das demências em idosos com idade superior a 65 anos no Brasil ${ }^{10}$, seguida pelas demências vasculares (DV), comumente associadas à demência de Alzheimer, constituindo a demência mista".

Com a evolução da doença, os idosos, em sua maioria residentes na comunidade, tornam-se progressivamente dependentes dos chamados "cuidadores"6,7, especialmente do cuidador principal, onerado não somente no que diz respeito à necessidade de assistência e atenção à saúde, como também às múltiplas tarefas práticas, à atenção e ao carinho dispensados ao doente, aos fatores econômicos, ao uso de transportes coletivos, à moradia, entre outros fatores, que tornam a tarefa do cuidador um desafio 6,12,13.

As sobrecargas física e psíquica a que os cuidadores de idosos com diagnóstico de demência estão expostos não raro leva à má qualidade de vida desses indivíduos ${ }^{5,8}$, com impacto negativo resultante da qualidade do próprio portador ${ }^{14,15}$

As consequências mais comuns do impacto de cuidar de um portador de demência são os problemas sociais, piora da saúde física e psíquica ${ }^{4,16}$, preditores para a institucionalização do indivíduo com demência já nos estágios iniciais ${ }^{4,17}$.

Considerando-se a importância de se conhecer a qualidade de vida dos cuidadores de idosos com demência, a fim de planejar intervenções voltadas à saúde deles, e a escassez de publicações, no que se refere à qualidade de vida destes cuidadores, sobretudo nos países em desenvolvimento, este trabalho tem como objetivo realizar revisão sistemática da literatura sobre a qualidade de vida em cuidadores de idosos portadores de demência de Alzheimer.

\section{MÉTODOS}

Foram analisados os artigos com os descritores quality of life, caregivers, Alzheimer disease na base de dados PubMed e MedLine, de 1997 a 2008. Foram encontrados 274 resultados, dos quais 16 foram incluídos. Artigos que não versavam especificamente sobre o tema referido foram excluídos. Além dos trabalhos encontrados na busca, também foram incluídas outras 39 publicações, referenciadas nos artigos, que foram consideradas importantes para o entendimento do presente tema.

\section{Qualidade de vida e demência}

O conceito de qualidade de vida é subjetivo, dependente do nível sociocultural, da faixa etária e das aspirações pessoais do indivíduo. A qualidade de vida está relacionada à autoestima e ao bem-estar pessoal, abrangendo uma série de aspectos, como a capacidade funcional, o nível socioeconômico, o estado emocional, a interação social, a atividade intelectual, o autocuidado, o suporte familiar, o próprio estado de saúde, os valores culturais, éticos e a religiosidade ${ }^{18}$. Também compreende o estilo de vida, a satisfação com o emprego e/ou com as atividades diárias e o ambiente em que se vive ${ }^{19-21}$.

A Organização Mundial da Saúde (OMS), a partir do início dos anos 1990, constatou que as medidas de qualidade de vida revestem-se de particular importância na avaliação de saúde, tanto dentro de uma perspectiva individual quanto social ${ }^{22}$

Neste contexto, uma das doenças mais impactantes é a demência, não somente pela ocorrência significativa ${ }^{6}$, como também pelos índices de morbidade preocupantes nos cuidadores e/ou familiares dos portadores dessa enfermidade ${ }^{4,7}$.

Os estudos epidemiológicos indicam que a prevalência da demência dobra a cada cinco anos entre as idades de 65 
e 85. Em pesquisa realizada em uma cidade do interior de São Paulo, verificou-se prevalência de demência entre 1,3\%, na faixa etária de 60 a 69 anos, a 36,9\%, para indivíduos com

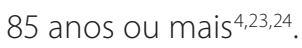

De acordo com o Relatório da OMS22, a demência contribui para $11,2 \%$ dos anos vividos com incapacidade nas pessoas com 60 anos ou mais, superior ao acidente vascular encefálico (9,5\%), à doença cardiovascular (5\%) e a todas as formas de câncer (2,4\%). O peso da incapacidade para demência, estimado pelo consenso especialista multidisciplinar e internacional, foi maior do que para quase todas as outras condições de saúde, exceto lesão medular e câncer em fase terminal. Sessenta por cento dos indivíduos com demência vivem em países em desenvolvimento, estimando-se que este aumento passará para 70\% em 2040, sendo esta taxa de aumento prevista para três a quatro vezes maior nas áreas em desenvolvimento, quando comparadas com as desenvolvidas ${ }^{3}$, o que torna esta questão importante para a saúde pública de países como o Brasil.

A principal causa de demência é a doença de Alzheimer, que corresponde a $60 \%$ de todos os $\operatorname{casos}^{25}$. Em estudo realizado na cidade de Catanduva (interior do estado de São Paulo) a demência de Alzheimer, isoladamente, foi responsável por 55,1\% dos casos de demência acima dos 64 anos de idade, enquanto a DA associada à doença cérebrovascular (demência mista), por 14,4\% $\%^{24,26}$.

\section{Cuidadores de portadores de demência de Alzheimer. Como está a qualidade de vida?}

Os idosos portadores de síndrome demencial vivem, em sua grande maioria, na comunidade. Com a evolução da doença, o idoso torna-se progressivamente dependente dos chamados "cuidadores" do Idoso (1999) como a pessoa, membro ou não da família, que cuida do idoso doente ou dependente no exercício das suas atividades diárias, como alimentação, higiene pessoal, além de outros serviços exigidos no cotidiano.

Em estudos realizados com cuidadores informais, verificou-se que a maioria reside com o idoso doente, são mulheres, filhas ou esposas, casados, com idade média entre 55 e 65 anos, sem emprego ou do lar, com oito ou mais anos de escolaridade ${ }^{4,27}$. As pesquisas na sua maioria são concordantes quanto ao sexo e ao grau de parentesco dos cuidadores 4,6,28,29. Em estudo italiano, 80\% a 90\% dos cuidadores eram familiares do idoso doente, sendo $70 \%$ do sexo feminino ${ }^{30}$.

Os cuidadores informais são divididos em cuidadores primários, secundários e terciários, segundo as tarefas realizadas, o seu grau de envolvimento com as tarefas desempenhadas e com as responsabilidades do cuidado. $O$ primeiro grupo, também denominado "cuidadores principais", é composto por aqueles que assumem a responsabilidade integral por supervisionar, orientar, acompanhar e/ou cuidar diretamente da pessoa idosa, ou seja, é o grupo que

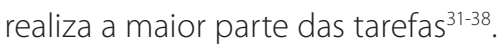

A demência, sabidamente, evolui em estágios, desde quadro incipiente até demência grave, o cuidador é obrigado a enfrentar diversas dificuldades? ${ }^{7}$ Cuidar de idoso portador de demência em casa apresenta vários desafios relacionados ao declínio progressivo na cognição e no comportamento de interação social ${ }^{39}$.

O cuidado com esse paciente pode ser experiência devastadora levando à piora da qualidade de vida de ambos os indivíduos. De acordo com a literatura, as más condições de saúde física e psíquica do cuidador são apontadas como um dos importantes preditores para institucionalização do indivíduo com demência já nos estágios iniciais ${ }^{4,17,40}$. Dessa forma, com o crescente aumento na prevalência da demência de Alzheimer e do número de cuidadores haveria elevação subsequente de custos em cuidados médicos, gerando problemas econômicos para a própria nação ${ }^{41}$.

Uma pesquisa recente avaliou o significado de qualidade de vida na visão do cuidador de portadores de demência de Alzheimer e procurou identificar fatores que alterassem a sua qualidade de vida, para melhor ou pior. Nesse estudo, os cuidadores associaram boa qualidade de vida ao bemestar psicológico, serenidade, bem-estar geral, estabilidade financeira, liberdade e tranquilidade; já em relação ao idoso cuidado, esses cuidadores informais referiram que fatores, como boa saúde e maior independência do idoso, associado a presença de alguém que prestasse auxilio aos cuidados, melhorariam a sua qualidade de vida. Por outro lado, fatores como preocupações sobre a evolução, prognóstico da doença e estresse diário tendem a piorar a qualidade de vida desses indivíduos ${ }^{41}$.

Diversos estudos investigaram fatores que melhorariam a qualidade de vida dos cuidadores. Um estudo encontrou que boa saúde física, envelhecimento saudável, boas condições financeiras eram bons preditores para boa qualidade de vida ${ }^{42}$. Outros estudos encontraram que a presença de uma rede de apoio social, boa saúde mental e física, somada ao bem-estar espiritual levaria à melhor qualidade de vida $^{42-46}$.

Alguns estudos investigaram o impacto na qualidade de vida dos cuidadores submetidos a tratamento e apoio de suporte com profissionais da saúde, foi visto melhor qualidade de vida naqueles cuidadores que eram submetidos a auxílio para modificação do cuidado com o idoso por meio de sessões de terapia ocupacional em casa ${ }^{42,47}$.

Diversas pesquisas identificaram fatores que pioravam a qualidade de vida do cuidador. Depressão, ansiedade e tensão são sintomas comuns entre os cuidadores de pacientes com doença de Alzheimer, e diversos estudos avaliaram como essas emoções afetam a qualidade de vida dos cuidadores, chegando à conclusão de que a depressão está 
intimamente relacionada com a solidão, a qualidade da relação entre o cuidador/idoso e a sua personalidade, cultura e nível de estresse ${ }^{48,49}$, afirmando a importância de maior atenção dos profissionais de saúde em relação à orientação e ao encaminhamento desses indivíduos a serviços que possam Ihe prestar atendimento e esclarecimento adequado, buscando melhora da qualidade de vida do cuidador e do idoso 48 .

Pesquisas indicam que cuidadores de idosos com demência relatam mais cansaço, desgaste, revolta, depressão e somatizações do que familiares de idosos sem distúrbios neurodegenerativos ${ }^{6,7,29,50}$.

A qualidade de vida foi avaliada como negativa em cuidadores de portadores de demência que apresentavam alterações psicológicas e comportamentais ${ }^{51,52}$ que, por sua vez, levam à piora da qualidade do próprio portador ${ }^{14}$. Estes dados foram confirmados em um estudo multicêntrico, no qual a qualidade de vida de cuidadores principais de idosos com demência também se relacionou à duração da evolução da doença, tendo-se verificado que as cuidadoras muIheres têm pior qualidade de vida e maior índice de depressão do que cuidadores homen ${ }^{15}$.

Andrieu et al..$^{29}$ avaliaram o impacto na qualidade de vida de cuidadores de pacientes com demência e chegaram à conclusão de que cuidadores de idosos muito dependentes tinham diminuição da qualidade de vida pelo fato de essa atividade ter restringido significativamente a interação social desses indivíduos.

Roig et al..28 concluíram que cuidadores mulheres, com idade maior que 56 anos, casadas, com filhos, nível socioeconômico baixo, escolaridade baixa e que cuidam de idosos em estágio avançado de demência experimentaram maior sobrecarga e, consequentemente, apresentaram piora significativa da sua qualidade de vida.

Argimon et al. ${ }^{53}$, levando-se em conta o sexo dos cuidadores, avaliaram o estado emocional, a saúde mental, as mialgias (doença física) e a capacidade física, e concluíram que a saúde mental, a estrutura emocional e a doença física são muito frequentemente afetadas, de modo negativo, em mulheres, enquanto nos homens cuidadores apenas a capacidade física encontrou-se significativamente prejudicada.

\section{DISCUSSÃO}

As sobrecargas física e psíquica a que os cuidadores de idosos com diagnóstico de demência estão expostos não raro levam à má qualidade de vida desses indivíduos ${ }^{5,8}$. Problemas sociais, piora da saúde física e sintomas psiquiátricos - principalmente depressão - são as consequências mais comuns do impacto de cuidar do portador de demência, motivo pelo qual estratégias de intervenção têm sido desenvolvidas no sentido de diminuir o impacto sofrido pe- los cuidadores informais ${ }^{4,16}$. Estes cuidadores são onerados de diferentes maneiras pela tarefa de cuidar de um idoso doente, não somente no que diz respeito à necessidade de assistência e atenção à saúde, como também às múltiplas tarefas práticas, à atenção e ao carinho dispensados, aos fatores econômicos, ao uso de transportes coletivos, à moradia, entre outros, $6,12,13$.

Revisando os estudos brasileiros realizados com cuidadores familiares de idosos, não somente demenciados, Santos $^{18}$ verificou que em todos eles os cuidadores familiares se ressentem pela falta de uma rede de suporte mais efetivo nas áreas da saúde e social, e carecem de treinamentos e orientações específicas para a realização dos cuidados no âmbito domiciliar.

Garrido e Menezes ${ }^{4}$ ressaltam a necessidade de haver mais estudos no Brasil acerca dos cuidadores, além de boa formação dos profissionais de saúde especializados nesta área e implantação de programas de orientação e apoio ao cuidador que envolvam a família, a comunidade e o estado.

\section{CONCLUSÃO}

Por essa revisão, pode-se observar a avaliação da qualidade de vida negativa em cuidadores de idosos com demência de Alzheimer. A necessidade de maiores abordagens sociais e profissionais aos cuidadores de pacientes demenciados é realidade evidente. $\mathrm{O}$ esclarecimento aos profissionais de saúde sobre aspectos da qualidade de vida desse cuidador ajuda a direcionar estratégias para a melhora e a manutenção da qualidade de vida desses indivíduos. Entretanto, são necessários mais estudos relacionados a esse tema, principalmente em relação a abordagens terapêuticas eficazes nesse universo cuidador/idoso.

\section{REFERÊNCIAS}

1. The WHOQOL Group. The World Health Organization quality of life assessment: position paper from the world health organization, 1995. Soc Sci Med. 1995;41:1403-9.

2. Fleck MPA, et al. Application of the portuguese version of the abbreviated instrument of quality life WH0Q0L-bref. Rev Saude Publica. 2000;34(2):178-83.

3. Ferri CP, Prince M, Brayne C, Brodaty H, Fratiglioni L, Ganguli M, et al. Global prevalence of dementia: a delphi consensus study. Lancet. 2005;366:2112-7.

4. Garrido R, Menezes PR. Impacto em cuidadores de idosos com demência atendidos em um serviço psicogeriátrico. Rev Saude Publica. 2004;38(6):835-41.

5. Vilela LP, Caramelli PA. Doença de Alzheimer na visão de familiares de pacientes. Rev Assoc Med Bras. 2006;52(3):148-52.

6. Silveira TM, Caldas CP, Carneiro TF. Caring for the highly dependent elderly in the community: a study on the main family caregivers. Cad Saude Publica. 2006;22(8):1629-38.

7. Garrido, R, Almeida, OP. Behavioural problems in patients with dementia and their burden on carers. Arq Neuropsiquiatr. 1999;57(2B):427-34.

8. Schulz R, Martire LM. Family caregiving of persons with dementia: prevalence, health effects, and support strategies. Am J Geriatr Psychiatry. 2004;12(3):240-9. 
9. Manual diagnóstico e estatístico de transtornos mentais: DSM-IV. 4. ed. texto revisado [DSM-IV-R]. Traduzido por Batista D. Porto Alegre: Artmed; 2002.

10. Lopes MA, Bottino CMC. Prevalência de demência em diversas regiões do mundo. Análise dos estudos epidemiológicos de 1994 a 2000. Arq Neuropsiquiatr. 2002;60:61-9.

11. Gallucci Neto J, Tamellini MG, Forlenza OV. Diagnóstico diferencial das demências. Rev Psiq Clín. 2005;32(3):119-30

12. Coen RF, O'Boyle CA, Coakley D, Lawlor BA. Individual quality of life factors distinguishing low-burden and high-burden caregivers of dementia patients. Dement Geriatr Cogn Disord. 2002;13(3):164-70.

13. Veras RP, Caldas CP, Dantas SB, Sancho LG, Sicsú B, Motta LBC. Avaliação dos gastos com 0 cuidado do idoso com demência/Family care for demented elderly individuals: cost analysis. Rev Psiquiatr Clin. 2007;34(1):5-12.

14. Banerjee S, Smith SC, Lamping DL, Harwood RH, Foley B, Smith P, et al. Quality of life in dementia: more than just cognition. An analysis of associations with quality of life in dementia. J Neurol Neurosurg Psychiatry. 2006 Feb;77(2):146-8.

15. Thomas P, Lalloué F, Preux PM, Hazif-Thomas C, Pariel S, Inscale R, et al. Dementia patients caregivers quality of life: the PIXEL study. Int I Geriatr Psychiatry. 2006;21(1):50-6.

16. Perren S, Schmid R, Wettstein A. Caregivers' adaptation to change: the impact of increasing impairment of persons suffering from dementia on their caregivers' subjective wellbeing. Aging Ment Health. 2006 Sep;10(5):539-48.

17. Pinquart M, Sörensen S. Helping caregivers of persons with dementia: which interventions work and how large are their effects? Int Psychogeriatr. 2006 Dec;18(4):577-95.

18. Santos SMA. 0 cuidador familiar de idosos com demências: um estudo qualitativo em famílias de origem nipo-brasileira e brasileira [tese]. Campinas: Universidade Estadual de Campinas; 2003

19. Velarde JE, Avila FC. Methods for quality of life assessment. Rev Salud Publica. 2002:44(4):349-61.

20. Bowling A, Gabriel Z, Dakes J, Dowding LM, Evans O, Fleissig A, et al. Let's ask them: a national survey of definitions of quality of life and its enhancement among people aged 65 and over. Int J Aging Hum Dev. 2003; 56(4):269-306.

21. Vecchia A, Roberta Dalla, et al. Qualidade de vida na terceira idade: um conceito subjetivo. Rev Bras Epidemiol. 2005; 8(3).

22. World Health Organization. World Health Report 2003 - shaping the future. Genebra: WHO; 2003 .

23. Jorm AF. The epidemiology of Alzheimer's disease and related disorders. London: Chapman and Hill; 1990

24. Herrera Jr E, Caramelli P, Silveira AS, Nitrini R. Epidemiologic survey of dementia in a community-dwelling Brazilian population. Alzheimer Dis Assoc Disord. 2002;16:103-8.

25. LoGiudice D. Dementia: an update to refresh your memory. Intern Med J. 2002;2:535-40.

26. Teixeira Jr AL, Caramelli P. Apatia na doença de Alzheimer/Apathy in Alzheimer's disease. Rev Bras Psiquiatr. 2005;28(3):238-41.

27. Badia Llach X, et al. Calidad de vida, tiempo de dedicación y carga percibida por el cuidador principal informal del enfermo de Alzheimer. Aten Primaria. 2004;34(4):170-7.

28. Roig MV, Abengozar MC, Serra E. La sobrecarga em los cuidadores principales de enfermos de Alzheimer. Anales de Psicología. 1998 Fev;14(2):215-27.

29. Andrieu S, Rive B, Guilhaume C, Kurz X, Scuvée-Moreau J, Grand A, et al. New assessment of dependency in demented patients: Impact on the quality of life in informal caregivers. Psychiatry Clin Neurosci. 2007 Jun;61(3):234-42.

30. Censis. La mente rubata. Alzheimer's: i. costi e i bisogni assistenziali di una malattia familiare Milano: Franco Angeli; 1999.

31. Silverstein $M$, Litwak EA. Task-specific typology of intergenerational family structure in later life. Gerontologist. 1993;33(2):258-64.

32. Carletti SMM, Rejani MI. Atenção domiciliária ao paciente idoso. In: Pappaleo Neto M. Gerontologia. São Paulo: Atheneu; 1996.
33. Kramer BJ. Gain in the Caregiving Experience: where are we? What next? Gerontologist. 1997;37(2):218-32

34. Mendes PMT. Cuidadores: heróis anônimos do cotidiano. In: Karsch UMS, organizador. EnveIhecimento com dependência: revelando cuidadores. São Paulo: EDUC; 1998. p. 171-97.

35. Gonçalves, LHT, Alvarez AM, Santos SMA. Os cuidadores leigos de pessoas idosas. In: Duarte YA0, Diogo MJD, organizadores. Atendimento domiciliar um enfoque gerontológico. São Paulo: Atheneu; 2000

36. Sommerhalder E, Néri AL. Avaliação subjetiva da tarefa de cuidar: ônus e benefícios percebidos por cuidadoras familiares de idosos de alta dependência. In: Yuaso D. Treinamento de cuidadores familiares de idosos de alta dependência em acompanhamento domiciliário [dissertação]. Campinas: Universidade Estadual de Campinas; 2001.

37. Neri AL, organizadora. Cuidar de idosos no contexto da família: questões psicológicas e sociais. Campinas: Alínea; 2001. p. 93-134.

38. Yuaso D. Treinamento de cuidadores familiares de idosos de alta dependência em acompanhamento domiciliário [dissertação]. Campinas: Universidade Estadual de Campinas; 2001.

39. Neri AL, Carvalho VAML. 0 bem-estar do cuidador: aspectos psicossociais. In: Freitas EV, Neri AL, Cançado FAX, Gorzoni ML, Rocha SM, organizadores. Tratado de geriatria e gerontologia. Rio de Janeiro: Guanabara Koogan; 2002. p. 778-90.

40. Morriss R, Rovner B, German P. Clinical and psychosocial variables associated with different types of behaviour problems in new nursing home admissions. Int I Geriatr Psychiatr. 1995;10:547-55.

41. Argimon JM, Limon E, Vila J, Cabezas. Health-related quality of life of caregivers as a predictor of nursinghome placement of patients with dementia. Alzheimer's Dis Assoc Disord. 2005;19:41-4.

42. Vellone E, Piras G, Talucci C, Cohen MZ. Quality of life for caregivers of people with Alzheimer's disease. J Adv Nurs. 2007;61(2):222-31.

43. Domico VD. The impact of social support and meaning and purpose in life on quality of life of spousal caregivers of persons with dementia. [dissertação]. Birmingham: The University of Alabama at Birmingham; 1997.

44. Coen RF, O'Boyle CA, Coakley D, Lawlor BA. Dementia carer education and patient behaviour disturbance. International J Geriatric Psychiatry. 1999;14:302-6.

45. Chappell NL, Reid RC. Burden and well-being among caregivers: examining the distinction. Gerontologist. 2002;42:772-80

46. Spurlock WR. Spiritual well-being and caregiver burden in Alzheimer's caregivers. Geriatr Nurs. 2005 May-Jun;26(3):154-61.

47. Gitlin LN, Hauck WW, Dennis MP, Winter L. Maintenance of effects of the home environmental skill-building program for family caregivers and individuals with alzheimer's disease and related disorders. J Gerontology Series A: Biological Sciences and Medical Sciences. 2005:60:368-74.

48. Beeson R, Horton-Deutsch S, Farran C, Neundorfer M. Loneliness and depression in caregivers of person with Alzheimer's disease or related disorders. Issues Mental Health Nurs. 2000;21:779-806

49. Clyburn LD, Stones MJ, Hadjistavropoulos T, Tuokk H. Predicting caregiver burden and depression in Alzheimer's disease. Journals of Gerontology. Series B. Psych Scienc Soc Scienc. 2000;55:S2-13.

50. Coen RF, Swanwick GR, O'Boyle C, et al. Behaviour disturbance and other predictors of carer burden in Alzheimer's disease. Int J Geriatr Psychiatry. 1997;12:331-6.

51. Takahashi M, Tanaka K, Miyaoka H. Depression and associated factors of informal caregivers versus professional caregivers of demented patients. Psychiatry Clin Neurosci. 2005:59(4):473-80

52. Zhang B, Mitchell SL, Bambauer KZ, Jones R, Prigerson HG. Depressive symptom trajectories and associated risks among bereaved Alzheimer disease caregivers. Am J Geriatr Psychiatry. 2008 Feb;16(2):145-55.

53. Argimon JM, Limon E, Vila J, Cabezas. Health-related quality of life in carers of patients with dementia. Fam Pract. 2004 Aug;21(4):454-7. 